\title{
Crystal structure of sodium ytterbium diphosphate, $\mathrm{NaYbP}_{2} \mathrm{O}_{7}$
}

\author{
M. Férid*.I, K. Horchani-Naifer ${ }^{\mathrm{I}}$ and M. Trabelsi-Ayedi ${ }^{\mathrm{II}}$ \\ I Institut National de Recherche Scientifique et Technique, Laboratoire des Matériaux, B.P. 95, 2050 Hammam-Lif, Tunisia \\ "Faculté des Sciences de Bizerte, Laboratoire de Physico-Chimie Minérale. 7021 Zarzouna Bizerte. Tunisia
}

Received August 6, 2004, accepted and available on-line November 13, 2004; CSD no. 409801

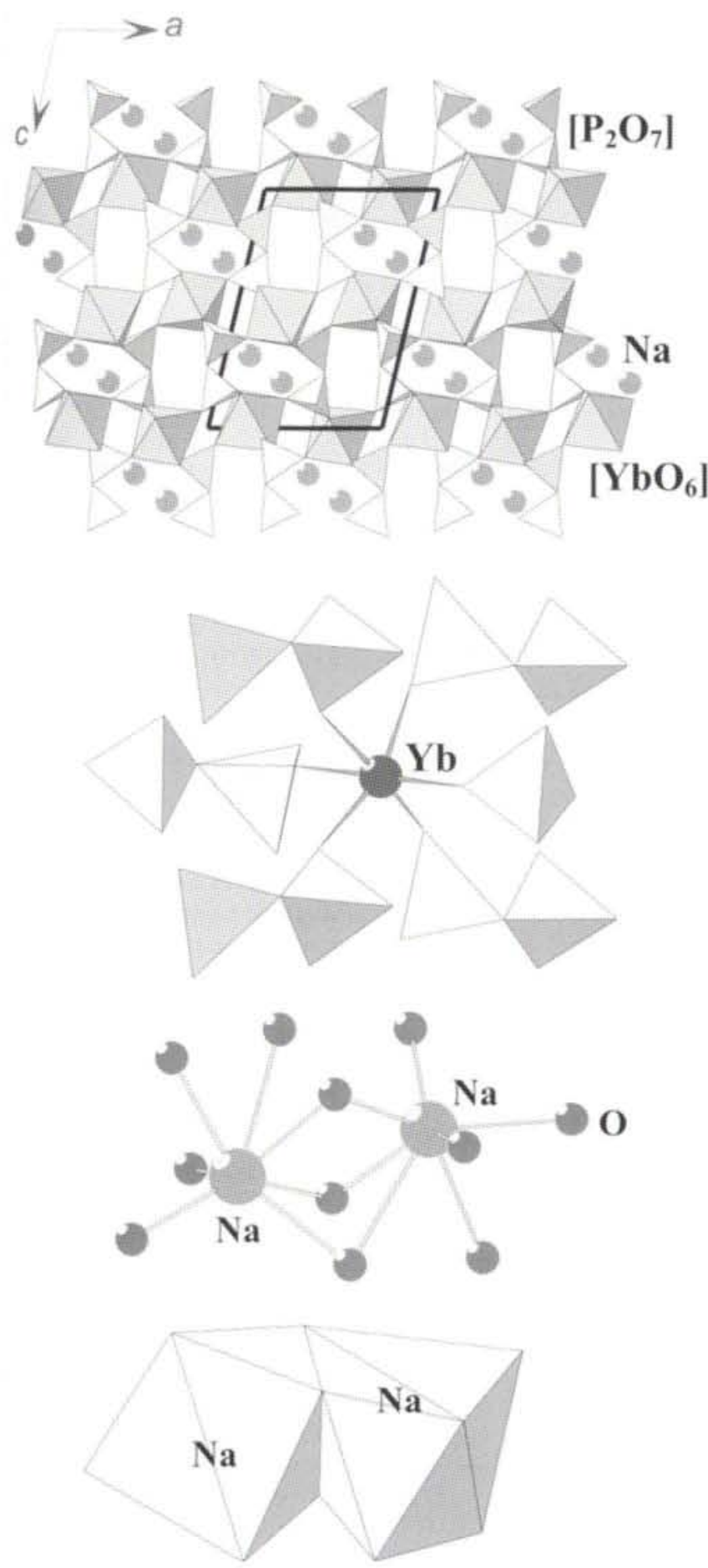

* Correspondence author (e-mail: mokhtar.ferid@inrst.rnrt.tn)

\author{
Abstract \\ $\mathrm{NaO} 7 \mathrm{P}_{2} \mathrm{Yb}$, monoclinic, $P 12_{1} / n 1$ (no. 14), $a=9.0211(3) \AA$, \\ $b=5.3571(4) \AA, c=12.7802(6) \AA, \beta=103.176(5)^{\circ}$, \\ $V=601.4 \AA^{3}, Z=4, R_{\text {gt }}(F)=0.040, w R_{\text {ref }}\left(F^{2}\right)=0.110$, \\ $T=293 \mathrm{~K}$.
}

\section{Source of material}

The $\mathrm{NaY}_{3} \mathrm{P}_{2} \mathrm{O}_{7}$ single crystals were grown. $3 \mathrm{~g}$ of $\mathrm{Na}_{3} \mathrm{P}_{3} \mathrm{O}_{9}$ and $0.5 \mathrm{~g}$ of ytterbium oxide $\mathrm{Yb}_{2} \mathrm{O}_{3}$ as starting materials were mixed. They were progressively heated in platinum crucible to $1273 \mathrm{~K}$ for twenty days, then cooled and maintained at $873 \mathrm{~K}$ for ten hours. The excess of $\mathrm{Na}_{3} \mathrm{P}_{3} \mathrm{O}_{9}$ was washed off with hot water and the crystals obtained were then isolated.

\section{Discussion}

The structure of $\mathrm{NaYbP}_{2} \mathrm{O}_{7}$ consists of $\mathrm{YbO}_{6}$ polyhedra and $\mathrm{P}_{2} \mathrm{O}_{7}{ }^{4-}$ diphosphate groups which lie in planes parallel to $(010)$, to form three-dimensional framework in which channels are noticed and where the sodium ions are located ( $C N 7)$. In $\mathrm{PO}_{4}$ tetrahedra, the $\mathrm{P}-\mathrm{O}$ distances vary from $1.481(6) \AA$ to $1.605(5) \AA$. The shortest distances correspond to the external $\mathrm{P}-\mathrm{O}$ connections, whereas largest of the distances $\mathrm{P}-\mathrm{O}$ are those of the bridge. The values of $\mathrm{O}-\mathrm{P}-\mathrm{O}$ angles vary in the interval $105.0(3)^{\circ}$ $113.8(3)^{\circ}$ and the angle of the $\mathrm{P}-\mathrm{O}-\mathrm{P}$ bridge has a value of $126.0^{\circ}$. These values are in conformity with the results found for the diphosphates [1]. Recently we have reported about the crystal structure of sodium and rare earth diphosphates such as $\mathrm{NaEuP}_{2} \mathrm{O}_{7}$ (monoclinic, space group $P 2_{1} / n$ ) [2] and $\mathrm{NaLaP}_{2} \mathrm{O}_{7}$ (orthorhombic, space group Pnma) [3]. The coordination polyhedron of the rare earth atoms in these diphosphates is not the same. In the case of the $\mathrm{NaLaP}_{2} \mathrm{O}_{7}$ and $\mathrm{NaEuP}_{2} \mathrm{O}_{7}$, lanthanum and europium cations are coordinated by nine and eight oxygen atoms, respectively, whereas in $\mathrm{NaYbP}_{2} \mathrm{O}_{7}$ the coordination number is six. The difference of the rare earth coordination can be simply explained by the size effect of the cations, but electronic configuration of the rare earth may also play an important role. $\mathrm{CsYbP}_{2} \mathrm{O}_{7}$ crystallizes in the same space group as $\mathrm{NaYbP}_{2} \mathrm{O}_{7}$ [4]. But both are not isotypic and have, e.g., completely different lattice parameters.

Table 1. Data collection and handling.

Crystal: Wavelength:

$\mu$ :

Diffractometer, scan mode: $2 \theta_{\max }$ :

$N(h k l)_{\text {measured, }} N(h k l)_{\text {unique: }}$

Criterion for $I_{\mathrm{obs}}, N(\mathrm{hkl})_{\mathrm{gt}}$ : $N(\text { param })_{\text {refined: }}$

Programs: colorless prism, size $0.10 \times 0.13 \times 0.30 \mathrm{~mm}$ Mo $K_{\alpha}$ radiation ( $0.71073 \AA$ ) $161.39 \mathrm{~cm}^{-1}$

Enraf-Nonius MACH 3, $\omega$ $59.98^{\circ}$

1819,1755

$I_{\text {obs }}>2 \sigma\left(I_{\text {obs }}\right), 1532$

101

SHELXS-97 [5], SHELXL-97 [6], WinGX [7], DIAMOND [8] 
Table 2. Atomic coordinates and displacement parameters (in $\AA^{2}$ ).

\begin{tabular}{|c|c|c|c|c|c|c|c|c|c|c|}
\hline Atom & Site & $x$ & $y$ & 2 & $U_{11}$ & $U_{22}$ & $U_{33}$ & $U_{12}$ & $U_{13}$ & $U_{23}$ \\
\hline $\mathrm{Yb}$ & $4 e$ & $0.76943(2)$ & $0.76384(4)$ & $0.02359(2)$ & $0.0062(2)$ & $0.0140(2)$ & $0.0038(2)$ & $0.00031(7)$ & $0.0000(1)$ & $0.00018(7)$ \\
\hline$P(1)$ & $4 e$ & $0.9716(2)$ & $1.2512(2)$ & $0.1536(2)$ & $0.0059(7)$ & $0.0135(7)$ & $0.0039(7)$ & $0.0000(4)$ & $0.0001(6)$ & $-0.0001(4)$ \\
\hline$P(2)$ & $4 e$ & $0.5682(2)$ & $0.2824(3)$ & $-0.1180(1)$ & $0.0065(7)$ & $0.0143(6)$ & $0.0032(7)$ & $-0.0002(5)$ & $-0.0011(5)$ & $0.0005(5)$ \\
\hline $\mathrm{Na}$ & $4 e$ & $0.6384(4)$ & $1.2280(5)$ & $0.1931(3)$ & $0.018(2)$ & $0.021(1)$ & $0.018(2)$ & $0.0018(9)$ & $0.002(1)$ & $-0.0005(9)$ \\
\hline$O(1)$ & $4 e$ & $1.0596(8)$ & $1.2587(8)$ & $0.0690(5)$ & $0.017(3)$ & $0.033(3)$ & $0.006(2)$ & $0.003(2)$ & $0.005(2)$ & $0.001(2)$ \\
\hline$O(2)$ & $4 e$ & $0.8908(5)$ & $1.0059(8)$ & $0.1594(3)$ & $0.015(2)$ & $0.014(2)$ & $0.005(2)$ & $-0.002(2)$ & $0.000(1)$ & $0.001(1)$ \\
\hline$O(3)$ & $4 e$ & $0.8605(5)$ & $1.4693(8)$ & $0.1458(3)$ & $0.013(2)$ & $0.016(2)$ & $0.006(2)$ & $0.005(2)$ & $0.000(1)$ & $0.002(2)$ \\
\hline$O(4)$ & $4 e$ & $1.0948(5)$ & $1.2793(8)$ & $0.2647(4)$ & $0.005(2)$ & $0.021(2)$ & $0.008(2)$ & $-0.001(1)$ & $0.001(2)$ & $0.002(1)$ \\
\hline$O(5)$ & $4 e$ & $0.6817(5)$ & $0.1251(9)$ & $-0.0422(3)$ & $0.022(2)$ & $0.022(2)$ & $0.009(2)$ & $0.002(2)$ & $-0.004(2)$ & $0.002(2)$ \\
\hline$O(6)$ & $4 e$ & $0.4051(6)$ & $0.2091(9)$ & $-0.1231(4)$ & $0.010(2)$ & $0.029(2)$ & $0.010(2)$ & $-0.003(2)$ & $0.007(2)$ & $-0.005(2)$ \\
\hline$\alpha(7)$ & $4 e$ & $0.5931(5)$ & $0.5593(8)$ & $-0.0968(3)$ & $0.016(2)$ & $0.014(2)$ & $0.010(2)$ & $-0.001(2)$ & $-0.003(2)$ & $0.002(2)$ \\
\hline
\end{tabular}

Acknowledgment. The authors thank Professor Mohamed Rzaigui for the data collection.

\section{References}

1. Durif, A.: Crystal chemistry of condensed phosphates. Plenum Press, New York 1995.

2. Ferid, M.; Horchani, K.; Amami, J.: Preparation, structure and infrared spectrum of $\mathrm{NaEuP}_{2} \mathrm{O}$. Mater. Res. Bull. 39 (2004) 1949-1955.

3. Ferid, M.; Horchani-Naifer, K.: Synthesis, crystal structure and vibrational spectra of a new form of $\mathrm{NaLaP}_{2} \mathrm{O}$. Mater. Res. Bull. 39 (2004) 2209-2217.

4. Jansen, M.; Wu, G. Q.; Königstein, K.: Crystal structure of caesium ytterbium diphosphate, Cs $\mathrm{CbP}_{2}$ O 7 . Z. Kristallogr. 197 (1991) 245-246.
5. Sheldrick, G. M.: SHELXS-97. Program for the Solution of Crystal Structures. University of Göttingen, Germany 1997.

6. Sheldrick, G. M.: SHELXL-97. Program for the Refinement of Crystal Structures. University of Göttingen, Germany 1997.

7. Farrugia, L. J.: WinGX suite for small-molecule single-crystal crystallography. J. Appl. Crystallogr. 32 (1999) 837-838.

8. Brandenburg K.: DLAMOND. Visual Crystal Structure Information System. Version 2.1e. Crystal Impact GbR, Bonn, Germany 2001. 